\title{
Knowledge and utilization of prevention of mother-to-child transmission of HIV services among pregnant women in Tanzania
}

Esther Ngadaya ${ }^{{ }^{*}}$ Angela E. Shija², Calvin Sindato ${ }^{3}$, Amos Kahwa', Godfather Kimaro', Mbazi Senkoro', Nicholaus P. Mnyambwa', Doreen Philibert', Togolai J Mbilu ${ }^{3}$, Celina Mandara ${ }^{4}$, Thomas Mwinyeheri', Justine Murashani" Aman K. Wilfred ${ }^{1}$ and Sayoki G. Mfinanga'

${ }^{1}$ National Institute for Medical Research Medical Research, Muhimbili Centre, Dar es Salaam, Tanzania.

${ }^{2}$ National Institute for Medical Research, Headquarters, Dar es Salaam, Tanzania.

${ }^{3}$ National Institute for Medical Research, Tabora Research Centre, Tanzania

${ }^{4}$ National Institute for Medical Research, Tanga Medical Research Centre, Tanzania

Abstract
Background: Human Immunodeficiency Virus (HIV) infection among children is mainly vectored through mother-to-child-transmission. Prevention of mother-to-child-transmission strategy is highly effective; however, its accessibility and utilization are affected by the lack of knowledge among other factors.

Methods: A cross-sectional study was conducted among antenatal care attendees in two districts in Tanzania to determine their knowledge and utilization of prevention of MTCT services.

Results: We interviewed 160 antenatal care attendees aged 18-45 years with a mean (SD) age of 30.4 (6.3) years; 74 (46.2 \%) were HIV-infected. HIV-infected women demonstrated significantly correct knowledge of HIV $(p=0.001)$ and AIDS $(p=0.014)$ than uninfected individuals. HIV-infected women also significantly demonstrated correct knowledge of mother-to-child-transmission during pregnancy than HIV-uninfected women $(p=0.016)$ and during delivery $(p=0.005)$. A significant proportion of HIV-positive women compared to HIV-negative women were aware that correct use of antiretroviral during pregnancy can reduce the risk of mother-to-child-transmission of HIV ( $p<0.039)$, but only $6(3.75 \%)$ of all women were aware that correct use of antiretroviral during delivery can significantly reduce the risk of mother-to-child-transmission. HIV-infected women had a significant comprehensive knowledge of HIV/AIDS ( $p=0.001)$ and prevention of mother-to-child-transmission of HIV $(p=0.006)$ than HIV-negative women. Comprehensive knowledge prevention of mother-to-child-transmission of HIV was low among the study participants. Male partner's involvement in maternal antenatal care was significantly higher among HIV-infected women than males from the HIV-infected women group $(p<0.006)$.

Conclusion: This study demonstrated inadequate knowledge on PMTCT among women who made ANC visits. HIV uninfected women had poorer knowledge compared to the HIV infected ones. Routine HIV counseling and testing services were highly accepted among these women.

Keywords: Prevention of mother-to-child-transmission (PMTCT); mother-to-child-transmission (MTCT); antenatal care (ANC); HIV/AIDS knowledge.

\section{Introduction}

Transmission of Human Immunodeficiency Virus (HIV) from an infected mother to her child during pregnancy, labour, delivery, or breastfeeding is known as mother-to-child transmission (MTCT) and it continues to be a major source of HIV infection among children (WHO, 2016). In 2010, it was estimated that only $45 \%$ of HIV-positive pregnant women in Sub-Saharan Africa had access to prevention of mother-to-child-transmission (PMTCT) services (WHO, 2007b). PMTCT has been at the front of global

\footnotetext{
*Corresponding author: Dr. Esther Ngadaya, MD, PhD (engadaya@yahoo.com)
} 
public health activities and has a potential to improve both child and maternal health (WHO, 2016). But due to a number of barriers to its implementation (Hardon et al., 2012; Kalembo et al., 2012; Merga et al., 2016), MTC transmission rates remain high in developing countries.

According to UNAIDS (United Republic of Tanzania | UNAIDS 2015, 2015), adult HIV prevalence in Tanzania in 2015 was estimated at 4.7 percent; nearly a fifth of all new HIV-infections was due to MTCT) (The united Republic of Tanzania|, 2014). Among newly HIV infected children, MTCT accounts for over 90 percent (De Cock et al., 2000). In the absence of PMTCT interventions, the overall rate of vertical HIV transmission ranges from 15-45 percent (WHO, 2016) with; 5-10\% infected during pregnancy; 10-15\% during childbirth and 5-20\% during breastfeeding (WHO, 2007a).

In the past 5 years, HIV prevalence in Tanzania among pregnant mothers attending for antenatal care (ANC) was $5.6 \%$ and that of exposed infants was $8.7 \%$ (Ministry of Health and Social Welfare, 2013), ranking Tanzania in the third position among countries with the highest proportion of HIV-infected children in sub-Saharan Africa (Foundation, n.d.). Such a transmission rate can be reduced to below 5\% with effective PMTCT interventions during pregnancy, childbirth (labour and delivery), and breastfeeding (WHO, 2016). Most of these interventions focus on antiretroviral prophylaxis for both women and infants, measures to prevent HIV acquisition in the pregnant women and safe childbirth practices, appropriate breastfeeding practices, HIV testing and other post-natal healthcare services (Padian et al., 2011).

Although PMTCT is the most effective strategy in preventing HIV infection from a mother to her newborn, its accessibility and utilization remain sub-optimal in most of the low-income countries like Tanzania. Accessibility and utilization of services in Tanzania are affected by the insufficient supply of ANC facilities, inadequate human resources, and barriers to accessing early infant diagnosis in up to $30 \%$ of facilities (UNAIDS, 2011). Poor knowledge, attitudes, socio-cultural issues, and access to the services have been reported to affect uptake and utilization of the services (Adedimeji et al., 2012; Falnes et al., 2010; Hembah-Hilekaan et al., 2012).

The effectiveness of PMTCT in Sub-Saharan Africa depends on male partners participation in the program (Kalembo et al., 2012; Nkuoh et al., 2010) as men are decision makers and providers of financial support for African families.

Comprehensive knowledge about HIV/AIDS is an indicator commonly used to measure knowledge potentials for HIV transmission and prevention. Although awareness of HIV/AIDS has been increasing in Tanzania, comprehensive knowledge, appropriate prevention, and non-stigmatizing behaviour have been lagging behind (NBS, 2011), and, as in other many resource-limited settings, PMTCT programs have not focused on HIV-negative women. Despite the critical need for HIV-positive pregnant women to understand mechanisms of HIV transmission and prevention to avoid or mitigate risks of infecting their neonates and/or sexual partners, the same knowledge is essential for HIVnegative women because the risk of acquiring HIV is increased during pregnancy and lactation (Gray et al., 2005; Mugo et al., 2011). This study was, therefore, conducted to assess the knowledge and utilization of PMTCT services of pregnant women (both positive and negative HIV/AIDS status) attending ANC in Mbulu and Mkuranga Districts, Tanzania.

\section{Materials and methods Study design}

We conducted a cross-sectional study using exit interviews (post-ANC service) with pregnant women aged 15-45 from selected health facilities in Mbulu and Mkuranga Districts in Tanzania. The study was conducted between 2011 and 2013. Exclusion criteria included a decline to participate in the study and deafness.

\section{Study sites}


This study was conducted in Mbulu District (Manyara Region) and Mkuranga District (Pwani Region). According to the Tanzania National Census (2012), Mbulu District had a population of 320,279 and Mkuranga had 222,921. Economically, Mkuranga residents are reliant on both fishing and farming, while farming is dominant in Mbulu. According to the 2011-2012 Tanzania Demographic and Health Survey (TDHS) (NBS, 2011), HIV prevalence among women of reproductive age (15-49 years) was $2.7 \%$ in Manyara Region and $9.2 \%$ in Pwani (Coast) Region.

\section{Sampling}

One district from each region (Manyara and Pwani) was randomly selected followed by the creation of a district-specific list of hospitals, health clinics, and dispensaries offering PMTCT. In each district, a district hospital was purposefully included in the study, as these are the major health care facilities serving as referral centres for the entire district.

An additional two facilities were also randomly selected to ensure representative sampling based on type (i.e., health centre and dispensary) and funder (i.e., government and faith-based). For Mbulu District, Hydom Referral Hospital (faith-based), Dongobesh Health Centre (government) and Masqaroda Dispensary (government) were selected. For Mkuranga District, Mkuranga District Hospital (government), St Vincent Dispensary (faith-based) and Kisiju Health Centre (government) were selected.

\section{Sample size estimation}

The sample size estimation was based on a single population proportion, computed using the online tool (Grady D, 1991). Using a proportion of 49\% pregnant women who were HIV-positive and receiving ARVs in public ANC clinics, a minimum sample size at $5 \%$ significance level was found to be 170 pregnant women. Allowing a non-response rate of $10 \%$, the adjusted sample size was 187 study participants.

\section{Data collection}

Exit interviews were conducted by nurses under the guidance of trained supervisors from the National Institute for Medical Research. A pre-tested structured questionnaire was used for data collection. The questionnaire mainly consisted of questions addressing socio-demographic characteristics, reproductive history, ANC visit, knowledge and utilization of PMTCT services. Most of the interview questions were adopted from 2011-2012 TDHS (NBS, 2011).

\section{Data management and analysis}

At the study site, forms were checked by supervisors for completeness and accuracy. The data were double entered and cleaned using Epidata ${ }^{\mathrm{TM}}$ Version 3.1 and SPSS Version 11.5. Logical checks were built into the database to further limit entry errors, and frequency runs were made for all variables to ensure the accuracy and consistency of the data outputs. Chi-square analysis of demographics, knowledge, and utilization of HIV/AIDS and PMTCT services were performed. Logistic regression to determine factors associated with dependent variables was performed. Dependent variables included knowledge of HIV/AIDS, MTCT, and PMTCT utilization. Independent variables were HIV status, age group, district, time to reach the clinic, payment for PMTCT services, number of ANC visits, HIV counseling, permission for HIV test, HIV test location, spousal awareness of HIV status, anticipated delivery site, marital status, education level and occupation.

\section{Criteria for knowledge assessment}

Knowledge on HIV and AIDS was categorized as "correct" if a woman knew that HIV is the state of having HIV infection and AIDS is a state of being sick due to opportunistic infection following HIV infection. Comprehensive knowledge of HIV/AIDS was present when a woman responded to the two components correctly.

Knowledge of MTCT of HIV was categorized as present if a woman mentioned that MTCT can occur in any of the following: during pregnancy, during delivery, or during breastfeeding. Comprehensive knowledge of MTCT of HIV was categorized as present if a woman mentioned all three transmission potentials. Likewise, knowledge of PTMCT of HIV was considered correct if a woman 
mentioned any of the following: mother using ARVs properly, appropriate provision of infant ARV prophylaxis, replacement feeding, or exclusive breastfeeding for the first six months and replacement feeding thereafter, and caesarean delivery. A woman was considered having comprehensive knowledge if she mentioned at least three out of the four above elements of PTMCT.

\section{Results}

\section{Socio-demographic characteristics}

Table 1 shows the socio-demographic characteristics of the study participants. Respondents' ages ranged from 18 to 45 years with a mean (SD) age of 30.4 (6.3) years. About two thirds of HIV infected individuals (62\%) and 53.5\% uninfected individuals were between 26 and 35 years. The majority of respondents (78\%) were living with their male partners; $60 \%$ had completed primary school or above, and 60.5 were self-employed. Although no statistical significance was found between the two groups for both education and employment status, living with their male partners was statistically significant $(p<0.004)$.

Table 1: Socio-demographic characteristics of the study participants

\begin{tabular}{lcccc}
\hline Demographics & $\begin{array}{c}\mathrm{HIV}+, \\
\mathrm{N}=74(46.2 \%)\end{array}$ & $\begin{array}{c}\text { HIV-, } \\
\mathrm{N}=86(53.8 \%)\end{array}$ & $\begin{array}{c}\text { Total; } \\
\mathrm{N}=160\end{array}$ & $p$ value \\
\hline Age & & & & \\
$18-25 \mathrm{y}$ & & & & \\
$26-35 \mathrm{y}$ & $10(13.5)$ & $28(32.6)$ & $38(23.8)$ & $*_{0} 0.012$ \\
$36-45 \mathrm{y}$ & $46(62.2)$ & $46(53.5)$ & $92(57.5)$ & \\
Marital status & $18(24.3)$ & $12(14.0)$ & $30(18.8)$ & \\
Living with a partner & & & & \\
Living alone & $50(67.6)$ & $75(87.2)$ & $125(78.1)$ & $* 0.004$ \\
Employment/occupation & $24(32.4)$ & $11(12.8)$ & $35(21.9)$ & \\
Non-employee & & & & \\
Employee & $23(31.5)$ & $31(36.9)$ & $54(34.4)$ & 0.397 \\
Self-employees & $4(5.5)$ & $4(4.8)$ & $8(5.1)$ & \\
Education Level & $46(63.0)$ & $49(58.3)$ & $95(60.5$ & \\
Never attended/completed primary school & $19(25.7)$ & $20(23.3)$ & $39(24.4)$ & 0.30 \\
Completed primary school & $47(63.5)$ & $49(57.0)$ & $96(60.0)$ & \\
Beyond primary school & $8(10.8)$ & $17(19.8)$ & $25(15.6)$ & \\
\hline
\end{tabular}

\section{Knowledge of HIV/AID and transmission}

Knowledge of transmission of MTCT of HIV among participants is shown in Table 2. Fifty (67.6\%) of the HIV infected individuals had the correct knowledge of HIV which differed significantly $(p=0.001)$ when compared to 28 (32.9\%) uninfected women. A higher percentage of HIV infected individuals were found to have correct knowledge of AIDs compared to un-infected individuals ( $58 ; 78.4 \%$ vs 51; 60.7\%; $\mathrm{p}=0.017)$. Likewise, HIV infected women as compared to HIV uninfected women were found to have correct knowledge of MTCT during pregnancy (36; 54.5\% vs 27; 36\%; $p=0.032$ ) and during delivery (42; $63.6 \%$ vs 30; 40.5\%; $p=0.006$ ).

Table 2: HIV/AIDS and MTCT Knowledge among study participants

\begin{tabular}{|c|c|c|c|c|}
\hline Variable & $\mathrm{HIV}+; \mathrm{N}=74 \quad \mathrm{n}(\%)$ & $\begin{array}{l}\text { HIV-; }=86 \\
n(\%)\end{array}$ & Total; $N=160(n \%)$ & p value \\
\hline
\end{tabular}




\begin{tabular}{|c|c|c|c|c|}
\hline Correct & $50(67.6)$ & $28(32.9)$ & $78(49.1)$ & *0.000 \\
\hline Incorrect & $24(32.4)$ & $57(67.1)$ & $81(50.9)$ & \\
\hline \multicolumn{5}{|c|}{ AIDS knowledge } \\
\hline Correct & $58(78.4)$ & $51(60.7)$ & $109(69.0)$ & *0.017 \\
\hline Incorrect & $16(21.6)$ & $33(39 \cdot 3)$ & $49(31.0)$ & \\
\hline \multicolumn{5}{|c|}{ Knowledge of HIV transmission to the child } \\
\hline Correct & $65(89.0)$ & $74(86.0)$ & $139(87.4)$ & 0.57 \\
\hline Incorrect & $8(11.0)$ & $12(14.0)$ & $20(12.6)$ & \\
\hline \multicolumn{5}{|c|}{ HIV transmission during pregnancy } \\
\hline Correct & $36(54.5)$ & $27(36.5)$ & $63(45.0)$ & $* 0.032$ \\
\hline Incorrect & $30(45 \cdot 5)$ & $47(63.5)$ & $77(55.0)$ & \\
\hline \multicolumn{5}{|c|}{ HIV transmission during delivery } \\
\hline Correct & $42(63.6)$ & $30(40.5)$ & $72(51.4)$ & $*_{0.006}$ \\
\hline Incorrect & $24(36.4)$ & $44(59 \cdot 5)$ & $68(48.6)$ & \\
\hline \multicolumn{5}{|c|}{ Knowledge of HIV transmission during breastfeeding } \\
\hline Correct & $51(77 \cdot 3)$ & $50(67.6)$ & 101(72.1) & \multirow[t]{2}{*}{0.201} \\
\hline Incorrect & $15(22.7)$ & $24(32.4)$ & $39(27.9)$ & \\
\hline
\end{tabular}

\section{Knowledge of prevention routes of $M T C T$}

Results in Table 3 shows that a significant proportion of HIV-positive women $43(65.2 \%)$ vs 29 (40\%) HIV-negative women were aware that correct use of ARV during pregnancy can reduce the risk of MTCT of HIV ( $p<0.039)$. Only 11 (12.2\%) women were aware that correct use of ARV's during delivery can reduce the risk of MTCT of HIV. Over three-quarters of the respondents were aware that given an ideal situation, abstention from breastfeeding can reduce the risk of MTCT of HIV, however, HIV infected women were more aware than HIV uninfected women ( $50 ; 75.8 \%$ vs $38 ; 55.1 \% ; p=0.01)$. Over 60 percent were aware of the protective effect of exclusive breastfeeding for six months, although, the awareness was higher among HIV infected individuals than that of HIV uninfected individuals (41; $62 \%$ vs $23 ; 33.3 \% ; p=0.001$ ).

Table 3: PMTCT Knowledge among study participants

\begin{tabular}{|c|c|c|c|c|}
\hline Demographics & $\mathrm{HIV}+; \mathrm{N}=74 \mathrm{n}(\%)$ & HIV-; $N=86(n \%)$ & Total; $N=160(n \%)$ & $\mathrm{p}$ value \\
\hline \multicolumn{5}{|c|}{ Knowledge of ARV use during pregnancy } \\
\hline Correct & $43(65.2)$ & $29(42.0)$ & $72(53.3)$ & $*_{0.07}$ \\
\hline Incorrect & $23(34.8)$ & $40(58.0)$ & $63(46.7)$ & \\
\hline \multicolumn{5}{|c|}{ Knowledge of ARV use during delivery } \\
\hline Correct & $8(12.1)$ & $3(4.4)$ & $11(8.2)$ & 0.10 \\
\hline Incorrect & $58(87.9)$ & $66(95.6)$ & $124(91.8)$ & \\
\hline
\end{tabular}




\begin{tabular}{|c|c|c|c|c|}
\hline Correct & $50(75.8)$ & $38(55.1)$ & $88(65.2)$ & $*_{0.01}$ \\
\hline Incorrect & $16(24.2)$ & $31(44.9)$ & $47(34.8)$ & \\
\hline \multicolumn{5}{|c|}{ Knowledge of exclusive breastfeeding } \\
\hline Correct & $41(62.1)$ & $23(33 \cdot 3)$ & $64(47.4)$ & $* 0.001$ \\
\hline Incorrect & $25(37.9)$ & $46(66.7)$ & $71(52.6)$ & \\
\hline
\end{tabular}

\section{Comprehensive knowledge}

In refence to Table 4, the majority of HIV infected women compared to HIV uninfected women had comprehensive knowledge of HIV/AIDS (48; 64.9\% vs 27; 31.4\%; $\mathrm{p}=0.001$ ); correct comprehensive knowledge of maternal to child transmission of HIV ( $53 ; 65 \%$ vs $34 ; 39 \% ; p=0.001$ ) and correct comprehensive knowledge of PMTCT of HIV (39; $48 \%$ vs 24; 28\%; $\mathrm{p}=0.006$ ). Generally, comprehensive knowledge of PMTCT of HIV was low among the study participants.

Table 4: Assessment of comprehensive knowledge among respondents

\begin{tabular}{|c|c|c|c|c|}
\hline & $\mathrm{HIV}+\mathrm{N}=74(\mathrm{n} \%)$ & HIV-: $N=86(n \%)$ & Total (n\%) & $P$ value \\
\hline \multicolumn{5}{|c|}{ HIV/AIDS knowledge } \\
\hline High & $48(64.9)$ & $27(31.4)$ & $75(46.9)$ & $*_{0.000}$ \\
\hline Low & $26(35.1)$ & $59(68.6)$ & $85(53.1)$ & \\
\hline \multicolumn{5}{|c|}{ MTCT knowledge } \\
\hline High & $53(65)$ & 34(39) & $87(50)$ & $*_{0.001}$ \\
\hline Low & $28(35)$ & $53(61)$ & $91(50)$ & \\
\hline \multicolumn{5}{|c|}{ PMTCT knowledge } \\
\hline High & $39(48)$ & $24(28)$ & $68(36)$ & $*_{0.006}$ \\
\hline Low & $42(52)$ & $63(72)$ & $115(63)$ & \\
\hline
\end{tabular}

\section{PMTCT utilization}

The majority (80\%) of women reported that daily activities did not prevent them from accessing PMTCT services. A total of 140 (91.5) women received HIV counseling (Table 5). Most of the HIV uninfected women (compared to infected women) had their first HIV test during ANC visit (67\% vs 29\%, $p<0.00$ ), while most of the HIV-positive women had their first test at Voluntary counselling and testing (VCT) centres ( $43 \%$ vs $9.7 \%, p<0.00$ ). The infected women significantly spend more money and time traveling to reach ANC that provides ARVs and sometimes due to fear of stigma.

Table 5: PMTCT Utilization among respondents

\begin{tabular}{|c|c|c|c|}
\hline Variable & HIV+ (n\%) & Total (n\%) & $p$ value \\
\hline
\end{tabular}




\begin{tabular}{|c|c|c|c|c|}
\hline Yes & $63(88.7)$ & $77(93.9)$ & $140(91.5)$ & 0.253 \\
\hline No & $8(11.3)$ & $5(6.1)$ & $13(8.5)$ & \\
\hline \multicolumn{5}{|c|}{ Tested for HIV during pregnancy } \\
\hline Yes & $72(100)$ & $85(100)$ & $157(98)$ & \\
\hline First tested at: ANC & $29(39.2)$ & $67(81.7)$ & $96(61.5)$ & $*_{0.00}$ \\
\hline VCT & $43(58.1)$ & $8(9.7)$ & $51(32.7)$ & \\
\hline Labour ward & $2(2.7)$ & $7(8.5)$ & $9(5.8)$ & \\
\hline \multicolumn{5}{|c|}{ Permission required for HIV testing } \\
\hline Yes & $20(28.2)$ & $35(42.7)$ & $55(36.0)$ & 0.062 \\
\hline No & $51(71.8)$ & $47(57 \cdot 3)$ & $98(64.1)$ & \\
\hline \multicolumn{5}{|c|}{ Number of ANC visits attended } \\
\hline$<=4$ visits & $16(24.0)$ & $22(27.0)$ & $38(25)$ & 0.675 \\
\hline$>4$ visits & $52(76.0)$ & $61(73.0)$ & $113(75)$ & \\
\hline \multicolumn{5}{|l|}{ Delivery site } \\
\hline Home & $7(10.1)$ & 13(16.9) & $20(13.7)$ & 0.200 \\
\hline Dispensary & $4(5.8)$ & $10(13.0)$ & $14(9.6)$ & \\
\hline Health centre & $5(7.2)$ & $7(9.1)$ & $12(8.2)$ & \\
\hline Hospital & $53(76.8)$ & $47(61.0)$ & $100(68.5)$ & \\
\hline
\end{tabular}

\section{Involvement of male partners in ANC}

A higher proportion of HIV infected women were accompanied by their male partners to ANC at least once during their pregnancy compared to HIV-infected women ( $66 \%$ vs $44 \%, p<0.006$ ). Generally, majority of the respondents were willing to inform their male partners about HIV testing. Among HIVpregnant women requiring permission from their male partners for HIV testing there was a threefold decrease of correct PMTCT knowledge compared with HIV- women not requiring permission (AOR:0.3; $\mathrm{Cl}$ :0.07-0.83). Among HIV-positive women lacking male partners' attendance during ANC visit there was a tenfold decrease in utilization of PMTCT services compared with HIV+ that have male partner's attendance (AOR:0.09; Cl:0.01-0.77).

\section{Discussion}

In this study, two-thirds of HIV-positive and HIV-negative pregnant women attending ANC the two hospitals in Tanzania demonstrated inadequate comprehensive knowledge of PMTCT of HIV. Routine HIV counseling and testing services were highly accepted among the two groups; HIV-infected and uninfected women. HIV-infected pregnant women had superior knowledge of PMCT compared to uninfected ones.

HIV knowledge observed in this study was generally low among all study participants, while relative higher among HIV-infected than uninfected pregnant women. The majority of women were 
aware of how HIV differs from AIDS and the potentials of MTCT of HIV. Less than a half women demonstrated an adequate comprehensive knowledge of MTC prevention while about one-third didn't know that abstention from breastfeeding for the first six months could lower the rate of HIV transmission to the baby, similar findings have been reported previously (Kourtis et al., 2007; Lunney et al., 2010).

None of the HIV-infected women could mention all the possible ways of preventing HIV transmission from the mother to her baby. Poor knowledge of PMTCT of HIV has been associated with poor utilization of the services (Falnes et al., 2010; hlarlaithe et al., 2014). This study raises a need for public interventions to improve understanding of MTCT in these communities with emphasize in rural areas around the country. The two districts are typical of many other places around the country that have similar geographical and demographic patterns that may encumber utilization of PMCT services.

The difference in the level of knowledge between HIV-infected and uninfected women was so significant to warrant an action. This anomaly may result in the HIV-positive pregnant women receive more in-depth counseling regarding HIV/AIDS, MTCT, and PMTCT compared to HIV-negative women, hence uninfected women lacking a proper understanding of the issues. It is time for the PMTCT programs to consider educating all women to adequately equip them with the salient PMTCT details needed for effective PMTCT program. This is because of the risk of acquiring new HIV infection increase during pregnancy and lactation (Gray et al., 2005; Mugo et al., 2011), and therefore at higher risk of infecting their children if they acquire HIV during this time. In Tanzania, the PMTCT services are provided under the ministry of health includes;- routine HIV testing and counseling, ARV treatment and prophylaxis for both mothers and children, safer delivery practices, counseling and support for safer infant feeding practices, long-term follow-up care for the mother-and-child and family planning (The united republic of Tanzania, n.d.).

Exclusive replacement (formula) feeding is the most widely used and effective method to prevent MTCT of HIV-1 through breastfeeding which is affordable, feasible, acceptable, sustainable, and safe (AFASS) (Kourtis et al., 2007; Oladokun et al., 2010). Over half of women, could not identify exclusive breastfeeding as one method of PMTCT and some HIV-positive women were breastfeeding their babies despite receiving counselling for safer feeding practices during their ANC visits; whether this was due to inadequacy education on PMTCT services or associated with fear of stigma or social norms could not be established by this study. This calls for more concerted efforts to raise public awareness on the importance of best breastfeeding practices. This study also reports nearly all of the respondents were not aware that ARV use during delivery can reduce MTCT.

In this study, we report a high utilization of HIV counseling and testing among ANC attendees and a large proportion of women delivered in health facilities with the assistance of health professionals. However, a significant proportion of HIV-infected women spent more money and time traveling a long distance to reach an ANC that provides ART and sometimes due to fear of social stigma, similar findings have been reported previously (Balasundaram et al., 2014)

Less than half of HIV-infected and two-thirds of uninfected women reported having been accompanied by their male partner at least once in their ANC. Poor involvement of men signifies that education on ANC is still not understood by communities. Low male participation in the PMTCT services has been previously reported to affect proper PMTCT utilization in the country (Msuya et al., 2008; Theuring et al., 2009). Although it was not established in this study; cultural issues, low knowledge, and social stigma have been reported to hindering ANCs attendance for men (Adedimeji et al., 2012; Falnes et al., 2010; Hembah-Hilekaan et al., 2012). Ideally, when a couple visits and attend ANCs activities together, make it easy for the couple to follow-up the advice they receive.

Limitation: Possible limitations of this study include lack of randomization to participants and possible biases like pressure from health workers and recall bias as participants were asked about prior 
pregnancy experience of PMTCT utilization. This study also did not access the impacts of other barriers (e.g., socio-cultural and financials barriers) towards accessibility and utilization of PMTCT services.

Conclusion: Generally, comprehensive knowledge of HIV prevention and transmission of HIV from MTC was low among ANC attendees and even much lower among HIV uninfected women compared to HIV infected women. This study raises a need for public interventions to improve understanding of MTCT in these communities

\section{Declarations:}

Ethics approval and consent to participate: The study protocol received ethical clearance from the Medical Research Coordinating Committee before it was implemented. Furthermore, all the fundamental ethical principles, including the respondents' written informed consent, risks, benefits and comfort of the respondents were followed according to the research ethical guidelines.

Consent to publish: Not applicable

Availability of data and materials: The datasets used and/or analyzed during the current study available from the corresponding author on reasonable request.

Competing interests: The authors declare no conflicts of interest.

Funding: This study received financial support from Global Fund Round 8 through Health Research users' trust fund of the National Institute for Medical Research, Tanzania. Additional funding was provided by the Welcome Trust to ESN and GSM for Manuscript development.

Authors' contribution: ESN is the principal author who was responsible for conceiving of the research idea, designing of the study, a collection of data, analysis, and interpretation of the results and writing of the draft and final manuscript. She is also the corresponding author. AS, CS, AK, GK, MS, NM, TM, CM, RS, TM, JM, AW, and SGM participated in proposal write up, data collection, data analysis and interpretation of the results, and writing of the draft and final manuscript.

Acknowledgments: Not applicable

\section{References}

Adedimeji, A., Abboud, N., Merdekios, B., \& Shiferaw, M. (2012). A Qualitative Study of Barriers to Effectiveness of Interventions to Prevent Mother-to-Child Transmission of HIV in Arba Minch, Ethiopia. International Journal of Population Research, 2012, 1-7. https://doi.org/10.1155/2012/532154

Balasundaram, A., Sarkar, S., Hamide, A., \& Lakshminarayanan, S. (2014). Socioepidemiologic Profile and Treatment-seeking Behaviour of HIV / AIDS Patients in a Tertiary-care Hospital in South India. 32(March 2012), 587-594.

De Cock, K. M., Fowler, M. G., Mercier, E., de Vincenzi, I., Saba, J., Hoff, E., Alnwick, D. J., Rogers, M., \& Shaffer, N. (2000). Prevention of mother-to-child HIV transmission in resource-poor countries: translating research into policy and practice. JAMA, 283(9), 1175-1182. http://www.ncbi.nlm.nih.gov/pubmed/10703780

Falnes, E. F., Tylleskar, T., de Paoli, M. M., Manongi, R., Engebretsen, I. M. S., E.F., F., T., T., M.M., D. P., \& R., M. (2010). Mothers' knowledge and utilization of prevention of mother to child transmission services in northern Tanzania. Journal of the International AIDS Society, 13(1), 36. https://doi.org/10.1186/1758-2652-1336

Foundation, T. | E. G. P. A. (n.d.). Country Spotlight: Tanzania. Retrieved April 12, 2017, from http://www.pedaids.org/countries/tanzania

Grady D, N. T. H. S. C. S. B. W. (1991). Sample size determization in health studies: A practical manual. WHO.

Gray, R. H., Li, X., Kigozi, G., Serwadda, D., Brahmbhatt, H., Wabwire-Mangen, F., Nalugoda, F., Kiddugavu, M., Sewankambo, N., Quinn, T. C., Reynolds, S. J., \& Wawer, M. J. (2005). Increased risk of incident HIV during pregnancy in Rakai, Uganda: a prospective study. The Lancet, 366(9492), 1182-1188. https://doi.org/10.1016/S0140-6736(05)67481-8

Hardon, A., Vernooij, E., Bongololo-Mbera, G., Cherutich, P., Desclaux, A., Kyaddondo, D., Ky-Zerbo, O., Neuman, M., Wanyenze, R., \& Obermeyer, C. (2012). Women's views on consent, counseling and confidentiality in PMTCT: a mixed-methods study in four African countries. BMC Public Health, 12(1), 26. 
https://doi.org/10.1186/1471-2458-12-26

Hembah-Hilekaan, S. K., Swende, T. Z., \& Bito, T. T. (2012). Knowledge, attitudes and barriers towards prevention of mother-to-child transmission of HIV among women attending antenatal clinics in Uyam District of ZakiBiam in Benue State, Nigeria. African Journal of Reproductive Health, 16(3), 28-35. http://www.ncbi.nlm.nih.gov/pubmed/23437497

hlarlaithe, M. O., Grede, N., de Pee, S., \& Bloem, M. (2014). Economic and Social Factors are Some of the Most Common Barriers Preventing Women from Accessing Maternal and Newborn Child Health (MNCH) and Prevention of Mother-to-Child Transmission (PMTCT) Services: A Literature Review. AIDS and Behavior, 18(S5), 516-530. https://doi.org/10.1007/s10461-014-0756-5

Kalembo, F. W., Yukai, D., Zgambo, M., \& Jun, Q. (2012). Male partner involvement in prevention of mother to child transmission of HIV in Sub-Saharan Africa: Successes, challenges and way forward. Open Journal of Preventive Medicine, 02(01), 35-42. https://doi.org/10.4236/ojpm.2012.21006

Kourtis, A. P., de Vincenzi, I., Taylor, A., Thigpen, M. C., Dao, H., Farley, T., \& Fowler, M. G. (2007). Prevention of human immunodeficiency virus-1 transmission to the infant through breastfeeding: new developments. American Journal of Obstetrics and Gynecology, 197(3), S113-S122. https://doi.org/10.1016/j.ajog.2007.03.003

Lunney, K. M., Iliff, P., Mutasa, K., Ntozini, R., Magder, L. S., Moulton, L. H., \& Humphrey, J. H. (2010). Associations between Breast Milk Viral Load, Mastitis, Exclusive Breast-Feeding, and Postnatal Transmission of HIV. Clinical Infectious Diseases, 21(3), 100201120631034-000. https://doi.org/10.1086/650535

Merga, H., Woldemichael, K., \& Dube, L. (2016). Utilization of Prevention of Mother-to-Child Transmission of HIV Services and Associated Factors among Antenatal Care Attending Mothers in Sebeta Town, Central Ethiopia. Advances in Public Health, 2016, 1-10. https://doi.org/10.1155/2016/6250898

Ministry of Health and Social Welfare. (2013). HIV / AIDS / STI Surveillance Report number 23.2013 (Issue 23).

Msuya, S. E., Mbizvo, E. M., Hussain, A., Uriyo, J., Sam, N. E., \& Stray-Pedersen, B. (2008). Low male partner participation in antenatal HIV counselling and testing in northern Tanzania: implications for preventive programs. AIDS Care, 20(6), 700-709. https://doi.org/10.1080/09540120701687059

Mugo, N. R., Heffron, R., Donnell, D., Wald, A., Were, E. O., Rees, H., Celum, C., Kiarie, J. N., Cohen, C. R., Kayintekore, K., Baeten, J. M., \& Partners in Prevention HSV/HIV Transmission Study Team. (2011). Increased risk of HIV-1 transmission in pregnancy: a prospective study among African HIV-1-serodiscordant couples. AIDS (London, England), 25(15), 1887-1895. https://doi.org/10.1097/QAD.ob013e32834a9338

NBS. (2011). Tanzania Demographic and Health Survey. https://dhsprogram.com/pubs/pdf/FR243/FR243\%5B24June2011\%5D.pdf

Nkuoh, G. N., Meyer, D. J., Tih, P. M., \& Nkfusai, J. (2010). Barriers to Men's Participation in Antenatal and Prevention of Mother-to-Child HIV Transmission Care in Cameroon, Africa. Journal of Midwifery \& Women's Health, 55(4), 363-369. https://doi.org/10.1016/j.jmwh.2010.02.009

Oladokun, R. E., Brown, B. J., Osinusi, K., Tiruneh, M., Semrau, K., Mwiya, M., Kasonde, P., Scott, N., Vwalika, C., Walter, J., Bulterys, M., Tsai, W., Thea, D., Mofenson, L., Miotti, P., Dransfield, K., Bray, D., Mmiro, F., \& Jackson, J. (2010). Infant-feeding pattern of HIV-positive women in a prevention of mother-to-child transmission (PMTCT) programme. AIDS Care, 22(9), 1108-1114. https://doi.org/10.1080/09540120903511008

Padian, N. S., McCoy, S. I., Karim, S. S. A., Hasen, N., Kim, J., Bartos, M., Katabira, E., Bertozzi, S. M., Schwartländer, B., \& Cohen, M. S. (2011). HIV prevention transformed: the new prevention research agenda. Lancet (London, England), 378(9787), 269-278. https://doi.org/10.1016/S0140-6736(11)60877-5

The united Republic of Tanzania|. (2014). Global response AIDS Response Country progress report. http://www.unaids.org/sites/default/files/country/documents/TZA_narrative_report_2014.pdf

The united republic of Tanzania, M. of H. and S. W. (n.d.). Prevention of Mother-to-Child Transmission of HIV. National guideline 2007.

Theuring, S., Mbezi, P., Luvanda, H., Jordan-Harder, B., Kunz, A., \& Harms, G. (2009). Male Involvement in PMTCT Services in Mbeya Region, Tanzania. AIDS and Behavior, 13(S1), 92-102. https://doi.org/10.1007/s10461-0099543-0

UNAIDS. (2011). 'Global plan towards the elimination of New HIV infections among children by 2015 and keeping their mothers alive".' http://www.unaids.org/sites/default/files/media_asset/20110609_JC2137_Global-PlanElimination-HIV-Children_en_1.pdf




WHO. (2007a). HIV transmission through breastfeeding A REVIEW OF AVAILABLE EVIDENCE. http://www.who.int WHO. (2007b). TOWARDS UNIVERSAL ACCESS Scaling up priority HIV/AIDS interventions in the health sector. www.who.int/hiv

WHO. (2016). Word Health Organization | Mother-to-child transmission of HIV. In World Health Organization. World Health Organization. http://www.who.int/hiv/topics/mtct/about/en/ 